\title{
Influência do gás de purga na prevenção da trinca de hidrogênio em juntas soldadas de um aço API $5 \mathrm{~L}$ X80
}

\author{
Roberta Adrielle Lima Vieira ${ }^{1 *}$ (1) \\ Waldemir dos Passos Martins ${ }^{1}$ \\ Valdemar Silva Leal ${ }^{1}$ \\ Antonio Ernandes Macêdo Paiva ${ }^{1}$
}

\section{Resumo}

O aumento da necessidade de aços utilizados em transporte de fluidos, como petróleo e gás natural, devido a novas reservas descobertas em ambientes adversos e maiores distâncias dos centros de consumo, gera um aumento progressivo por aços de alta resistência mecânica. Dessa forma, vêm sendo utilizados os aços ARBL (alta resistência e baixa liga), por apresentarem boa soldabilidade e resistência mecânica. Entretanto, esses aços tendem a apresentar problemas de soldabilidade em virtude do surgimento de trincas de hidrogênio $(\mathrm{TH})$. O objetivo deste estudo é avaliar a influência do gás de purga na prevenção do surgimento desse tipo de trinca em um aço API 5L X80, utilizando o teste implante acoplado a um sistema de emissão acústica (SEA) como forma de investigar a formação da TH. O processo de soldagem utilizado foi o FCAW-S e como metal de adição o arame tubular autoprotegido E71T-11, com e sem gás de purga. Os resultados evidenciaram que o gás de purga não é eficiente na prevenção da trinca de hidrogênio nas condições previstas neste trabalho e o teste implante acoplado ao SEA se mostrou uma ferramenta eficaz na detecção do momento de formação e propagação da trinca.

Palavras-chave: Trinca de hidrogênio; Gás de purga; Teste implante; Sistema de Emissão Acústica.

\section{Influence of the purge gas in the prevention of hydrogen cracking in welded joints of API $5 \mathrm{~L} \mathrm{X80}$ steel}

\begin{abstract}
The increased demand for steel used in the transportation of fluids, such as oil and natural gas, due to new reserves discovered in adverse environments and greater distances from consumption centers, generates a progressive increase in steel with high mechanical resistance. Thus, HSLA steels (high strength and low alloy) have been used as they present good weldability and mechanical resistance. However, these steels tend to present problems of weldability due to the emergence of Hydrogen Cracking (HC). This study aims to evaluate the influence of the purge gas in preventing the appearance of this type of cracking in an API 5L X80 steel, using the implant test coupled to an acoustic emission system (AES) as a way to investigate the formation of HC. The welding process used was the FCAW-S and the self- shielded tubular wire E71T-11 as the filler metal, with and without purge gas. The results showed that the purge gas is not efficient in preventing Hydrogen Cracking under the conditions provided for in this study, and the implant test coupled to the AES proved to be an effective tool in detecting the moment of the cracking formation and propagation.
\end{abstract}

Keywords: Hydrogen cracking; Purge gas; Implant test; Acoustic Emission System.

\section{Introdução}

Para favorecer a crescente demanda de energia, oleodutos são implantados no transporte de petróleo e gás natural para longas distâncias [1]. Assim, aços de alta resistência, os chamados ARBL (aço de alta resistência e baixa liga), estão sendo muito utilizados por razões econômicas, pois possuem melhores propriedades mecânicas e, consequentemente, tubulações com menores espessuras.

Um problema que os aços ARBL soldados podem sofrer são as chamadas trincas de hidrogênio (TH), formadas nas juntas soldadas desse material e ocupam um lugar de destaque

${ }^{1}$ Departamento de Mecânica e Materiais, Instituto Federal do Maranhão - IFMA, São Luís, MA, Brasil.

*Autor correspondente: roberta_adrielle15@hotmail.com

2176-1523 (C) 2021. Vieira et al. Publicado pela ABM. Este é um artigo publicado em acesso aberto (Open Access) sob a licença Creative Commons Attribution, que permite uso, distribuição e reprodução em qualquer meio, sem restrições desde que o trabalho original seja corretamente citado. 
nos problemas metalúrgicos, visto que a susceptibilidade para tal trinca aumenta com o aumento da resistência mecânica desses tipos de aços [2]. Essas trincas ocorrem em razão da presença de hidrogênio atômico, microestrutura susceptível e tensão. Durante o ciclo de resfriamento, após o processo de soldagem, as regiões austenitizadas passam por mudanças granulométricas e de transformação em microconstituintes diferentes de acordo com a taxa de resfriamento da solda $[3,4]$.

Segundo Ramos e Guilherme [5], para a soldagem de tubulação, faz-se necessário o uso de um gás de purga, de forma a proteger a poça de fusão na face interna do tubo e obter uma superfície adequada para aplicações que requerem controle de contaminantes presentes. Nesse tipo de proteção, são geralmente empregados os gases hélio, argônio e nitrogênio, ou misturas destes [6]. No estudo de Lagos et al. [7], para evitar a perda de qualidade da junta soldada do aço inoxidável AISI 304, foi utilizado o argônio como gás de purga para impedir a entrada do oxigênio atmosférico no cordão de solda.

A trinca de hidrogênio é um grave problema na soldagem dos aços de alta resistência, pois seu início e propagação ocorrem após a solidificação e resfriamento da solda [8]. Para analisar a susceptibilidade de um material para a formação de tal trinca quando soldado, a utilização de ensaios desenvolvidos tem facilitado a otimização de procedimentos de soldagem, de modo a favorecer soldas isentas ou com quantidades desprezíveis de trincas de hidrogênio [2].

O teste Implante, objeto deste trabalho, é um ensaio de restrição externa. Grande parte desses tipos de testes foram estabelecidos para que a trinca de hidrogênio aconteça na zona termicamente afetada (ZTA) [9]. Contudo, apenas o uso dos testes de susceptibilidade existentes não são garantias que suas respostas se estendam a uma aplicação prática real.

Tendo em vista essa questão, é necessário utilizar outra ferramenta vinculada ao teste para obter mais informações relevantes. Uma alternativa é o uso da técnica de Emissão
Acústica (EA) [4], cujo monitoramento é feito baseado na geração de ondas de emissão acústica para detecção do instante de formação e propagação da trinca $[3,10]$.

Nesse contexto, para obter resultados mais precisos quanto à influência do gás de purga na prevenção da $\mathrm{TH}$, buscou-se apresentar um estudo utilizando um sistema de Emissão Acústica acoplado ao teste Implante a fim de investigar a formação da trinca de hidrogênio na junta soldada em ensaios com e sem gás de purga.

\section{Materiais e métodos}

Para o ensaio implante, foram utilizadas chapas de aço API 5L X80 como metal de base (MB). A composição química e as propriedades mecânicas do MB são dadas na Tabela 1.

Como metal de adição, foi utilizado o arame tubular autoprotegido de diâmetro 1,6 mm, do tipo E71T-11. A composição química e as propriedades mecânicas do metal de adição são apresentadas na Tabela 2. O carbono equivalente apresentado nas Tabelas 1 e 2 foi determinado pela Equação 1 de Ito-Bessyo e Equação 2 do International Institute of Welding (IIW).

$$
\begin{aligned}
& \mathrm{Ceq}(\mathrm{Pcm})=\mathrm{C}+\frac{\mathrm{Si}}{30}+\frac{\mathrm{Mn}}{20}+\frac{\mathrm{Cu}}{20}+\frac{\mathrm{Ni}}{60}+\frac{\mathrm{Cr}}{20}+\frac{\mathrm{Mo}}{15}+\frac{\mathrm{V}}{10}+5 \mathrm{~B} \\
& \mathrm{Ceq}(\mathrm{IIW})=\mathrm{C}+\frac{\mathrm{Mn}}{6}+\frac{(\mathrm{Cr}+\mathrm{Mo}+\mathrm{V})}{5}+\frac{(\mathrm{Ni}+\mathrm{Cu})}{15}
\end{aligned}
$$

A Equação 1 é usada para aço com teores de carbono abaixo de $0,16 \%$ e microligados e é recomendado um valor de Ceq $\left(\mathrm{P}_{\mathrm{CM}}\right) \leq 0,25 \%$ para obter melhores resultados de soldabilidade e minimizar a ocorrência de trinca. A Equação 2, adotada pelo Instituto Internacional de Soldagem, é utilizada para aços com alto teor de carbono $(\mathrm{C}>0,12 \%)$ e tem ampla

\begin{tabular}{|c|c|c|c|c|c|c|c|c|c|}
\hline \multicolumn{10}{|c|}{ Composição química (\% em peso) } \\
\hline $\mathbf{C}$ & Mn & $\mathbf{S i}$ & $\mathbf{N b}$ & $\mathbf{V}$ & Ti & $\mathbf{C r}$ & $\mathbf{S}$ & \multicolumn{2}{|c|}{ Al } \\
\hline 0,05 & 1,81 & 0,29 & 0,069 & 0,033 & 0,02 & 0,17 & 0,001 & \multicolumn{2}{|c|}{0,02} \\
\hline $\mathrm{Cu}$ & $\mathrm{Ni}$ & Mo & $\mathrm{Sn}$ & $\mathrm{P}$ & $\mathrm{B}$ & $\mathrm{Ca}$ & As & \multicolumn{2}{|c|}{$\mathrm{N}$} \\
\hline 0,02 & 0,01 & 0,21 & 0,001 & 0,017 & 0,0002 & 0,0012 & 0,003 & \multicolumn{2}{|c|}{0,0034} \\
\hline \multicolumn{8}{|c|}{ Propriedades Mecânicas } & \multicolumn{2}{|c|}{ Carbono Equivalente } \\
\hline \multicolumn{2}{|c|}{ LE (MPa) } & \multicolumn{2}{|c|}{ LR (MPa) } & \multicolumn{2}{|c|}{$\varepsilon(\%)$} & \multicolumn{2}{|c|}{ HV } & $\begin{array}{c}\text { Ceq } \\
(\text { Pcm) }\end{array}$ & $\begin{array}{c}\text { Ceq } \\
\text { (IIW) }\end{array}$ \\
\hline \multicolumn{2}{|c|}{$550-660$} & \multicolumn{2}{|c|}{$631-741$} & \multicolumn{2}{|c|}{26} & \multicolumn{2}{|c|}{$225-250$} & 0,18 & 0,44 \\
\hline
\end{tabular}

Tabela 1. Composição química e propriedades mecânicas do aço API X80*

*Nota: Composição química, LE (Limite de Escoamento), LR (Limite de Resistência), $\varepsilon$ (Alongamento) e HV (Dureza) fornecidos pelo fabricante.

\begin{tabular}{|c|c|c|c|c|c|c|c|c|}
\hline \multicolumn{4}{|c|}{ Composição química (\% em peso) } & \multicolumn{3}{|c|}{ Propriedades Mecânicas } & \multicolumn{2}{|c|}{ Carbono Equivalente } \\
\hline $\mathbf{C}$ & Mn & $\mathbf{S i}$ & Al & LE (MPa) & LR (MPa) & $\varepsilon(\%)$ & Ceq (Pcm) & Ceq (IIW) \\
\hline 0,25 & 0,7 & 0,4 & 1,6 & 500 & 630 & 23 & 0,29 & 0,36 \\
\hline
\end{tabular}

Tabela 2. Composição química e propriedades mecânicas do metal de adição*

*Nota: Composição química, LE (Limite de Escoamento), LR (Limite de Resistência) e $\varepsilon$ (Alongamento) obtidos no Catálogo do Fabricante. 
aplicabilidade na indústria. Valores de Ceq (IIW) $\leq 0,45 \%$ indicam uma boa soldabilidade dos aços [4,11].

Os implantes (corpos de prova) foram retirados na direção da laminação e preparados conforme a norma AFNOR NF A 89-100 [12]. A justificativa para o uso do mesmo material na confecção dos implantes e chapas teste se deve pela necessidade da não variação da condutividade térmica.

A chapa teste foi usinada para a remoção da camada de óxido superficial e foram feitos dois (2) furos igualmente espaçados, com 6,35 mm e 2,5 mm de diâmetro, para a fixação do implante e termopar (este último para monitoração da temperatura de colocação do sensor de EA), respectivamente, conforme Figura 1a. A ilustração do implante, com as dimensões padronizadas, é apresentada na Figura 1b.

\subsection{Sistema de emissão acústica}

Para analisar os sinais emitidos pela trinca, foi utilizado um sistema de emissão acústica (SEA), composto por placa de aquisição de dados (A/D), sensor e interface computacional, apresentado na Figura 2a. A placa de aquisição de dados converte o sinal de saída do circuito retificador de onda de sua forma analógica para digital. O sensor para detecção das trincas modelo WD-FT64 converte as vibrações da superfície, causadas por uma onda elástica, em um sinal elétrico que é processado pelo SEA [4].
O Analisador de Emissão Acústica é o programa computacional, visto na Figura $2 b$, desenvolvido especificamente para esse sistema, que foi usado para capturar e armazenar os dados com um limite de corte de sinal previamente estabelecido.

$\mathrm{O}$ sistema trabalha na faixa de 0 a $10 \mathrm{~V}$ e o corte de ruído, após testes preliminares, baseado na metodologia adotada por Martins [4], foi de 0,32 V. A taxa de aquisição foi de 2000 pontos/s para todos os corpos de prova ensaiados e os sinais registrados se encontraram no mínimo de $0,32 \mathrm{~V}$.

\subsection{Ensaio Implante}

Para a realização dos ensaios, utilizou-se um equipamento para o ensaio implante que tem por finalidade aplicar uma carga ao implante impondo a condição de tensão interna. $\mathrm{O}$ equipamento utilizado é composto por dois tipos de sistemas: de tração (composto por um sistema hidráulico) e de monitoramento. Suas funções baseiam-se em tracionar o corpo de prova e monitorar o alívio de tensões, assim como a eventual ocorrência de trinca. A Figura 3a ilustra o equipamento do teste implante do Laboratório de Soldagem - LTPSolda/ DMM/IFMA. O ensaio fundamenta-se em inserir o Implante, fixado no suporte por rosqueamento, em um furo presente no tampo do equipamento para o ensaio e, posteriormente, a chapa com o furo. Após seu posicionamento no furo da

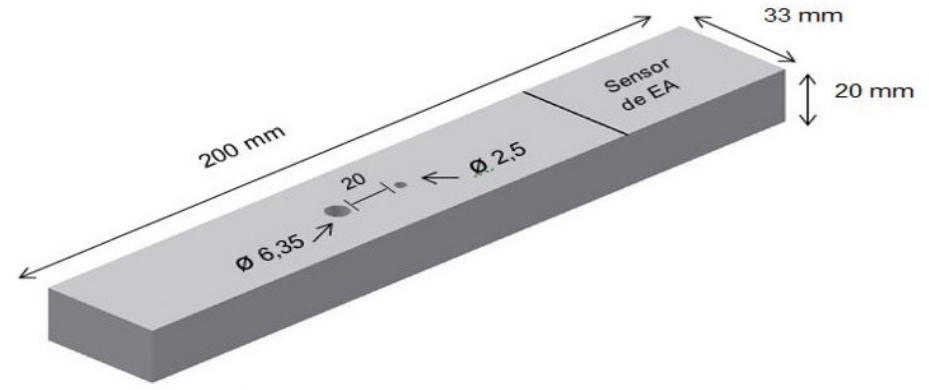

(a)

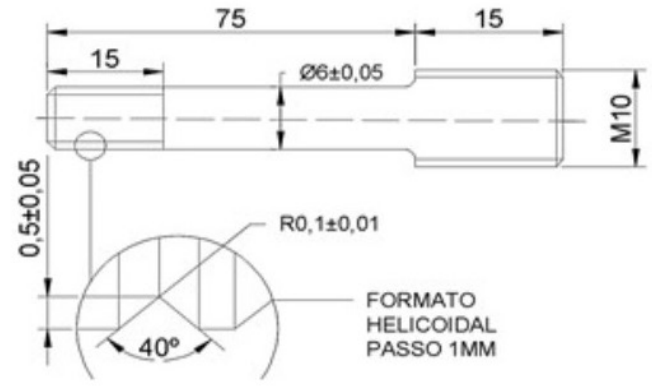

(b)

Figura 1. (a) Representação esquemática da chapa com o furo para colocação do implante e do termopar; (b) Dimensões do implante [4].

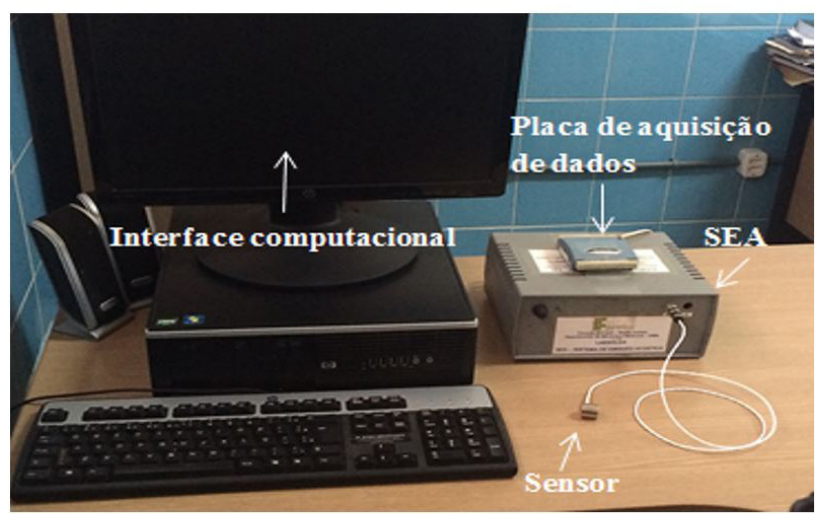

(a)

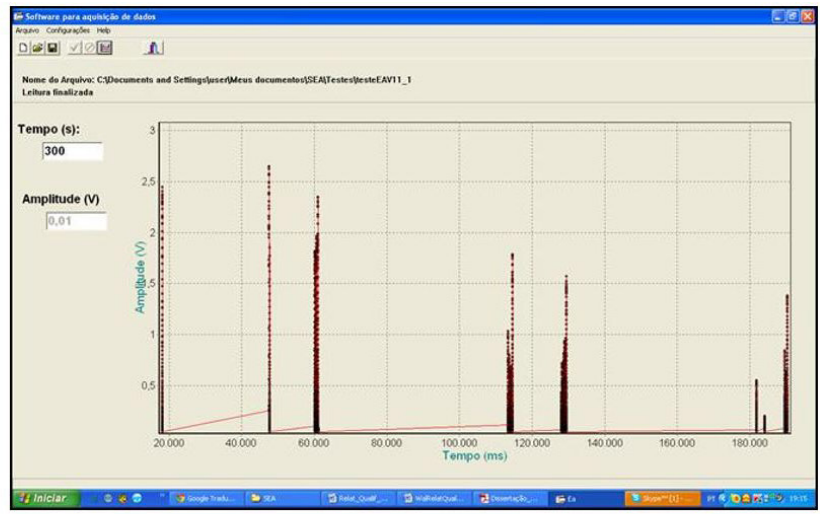

(b)

Figura 2. (a) Equipamentos que formam o Sistema de emissão acústica - SEA; (b) Analisador de sinais de EA. 
chapa, a parte entalhada do implante é nivelada com a sua superfície. Após essa montagem, foi feito um passe de solda de $100 \mathrm{~mm}$ sobre a chapa, passando por cima do furo e do corpo de prova (CP).

Os ensaios foram feitos em condições predeterminadas, utilizando o processo de soldagem e o consumível escolhido. As soldagens foram realizadas na condição com e sem gás de purga, sendo utilizado como gás de purga o argônio na vazão de $2 \mathrm{l} / \mathrm{min}$. O gás de purga foi injetado durante a soldagem, permanecendo na junta soldada até atingir a temperatura ambiente. A Figura 3b ilustra esquematicamente o teste implante com o furo de entrada do gás de purga.

Após a realização da soldagem e antes que o resfriamento completo da solda acontecesse, a uma temperatura de $150{ }^{\circ} \mathrm{C}$ (medida a partir do termopar instalado em furo próximo a ZTA), foi retirada a carga do sistema hidráulico, sendo então a tração gradativamente aplicada sobre o Implante. Dessa forma, o CP passou a ser submetido a um esforço de tração constante durante todo o ensaio.

A carga de tração constante foi medida a partir da célula de carga modelo TSA, conforme Figura 4a. O transmissor de pesagem recebeu o sinal da célula de carga e o transmitiu para a interface analógico/digital, mostrando a intensidade da solicitação num dado instante por meio de um indicador digital - Figura 4b.

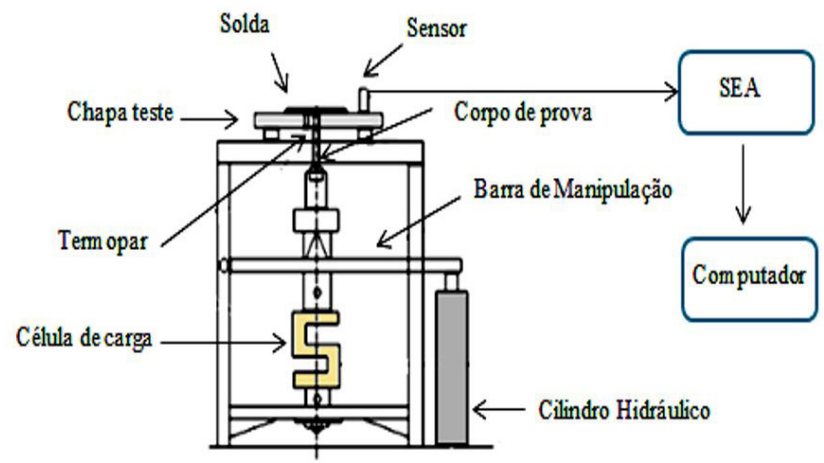

(a)
Quando a solda atingiu a temperatura pretendida, foi colocado o sensor de EA na chapa teste durante o ensaio e, então, foi iniciada a aquisição do sinal por 24 horas.

\subsection{Análise dos corpos de prova}

Os Implantes ensaiados foram analisados com os resultados apresentados pelo SEA. A finalidade do gás de purga é verificar sua contribuição na prevenção do fenômeno da TH. Neste estudo, foram considerados Implantes soldados com e sem gás de purga para diferentes energias de soldagem.

\section{Resultados e discussão}

Os resultados dos ensaios realizados demonstraram a viabilidade e sensibilidade do SEA na detecção e propagação da trinca de hidrogênio e foram confrontados com as micrografias e microdurezas dos corpos de prova ensaiados.

\subsection{Sinais do SEA}

$\mathrm{Na}$ Tabela 3, podem ser vistos os resultados dos ensaios com diferentes energias de soldagem e cargas aplicadas, onde a variável relacionada ao teste implante é

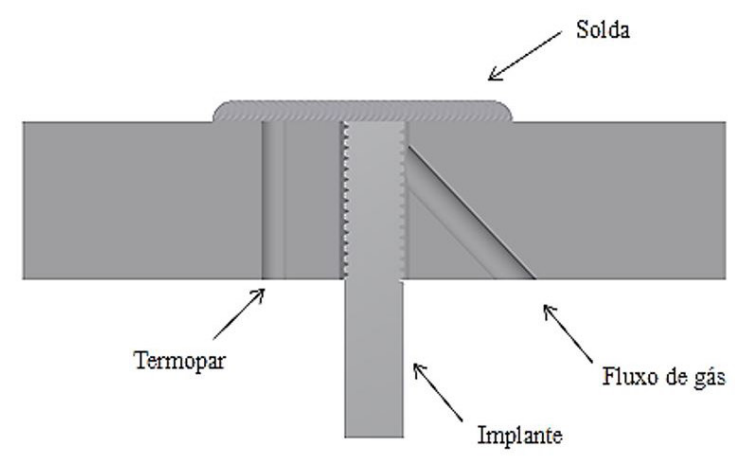

(b)

Figura 3. (a) Equipamento utilizado para ensaio implante LTPSolda/DMM/IFMA; (b) Chapa teste para ensaios que utilizaram gás de purga.

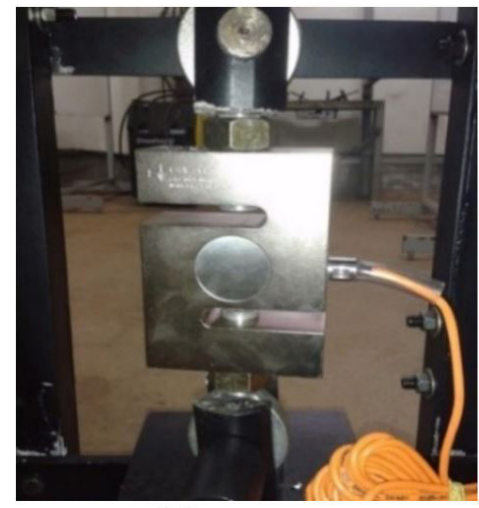

(a)

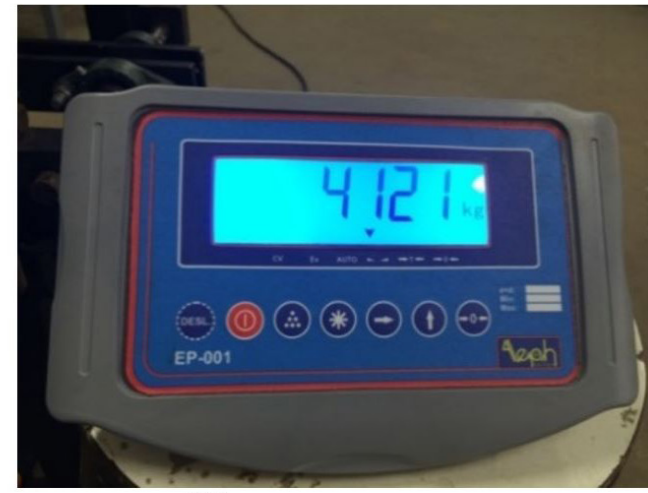

(b)

Figura 4. (a) Célula de carga modelo TSA; (b) Transmissor de pesagem que recebeu o sinal da célula de carga. 
Tabela 3. Resultados dos sinais de EA*

\begin{tabular}{|c|c|c|c|c|c|c|c|c|}
\hline Ensaio & Condição & $\begin{array}{c}E \\
(\mathrm{~kJ} / \mathrm{cm})\end{array}$ & $\begin{array}{c}\mathrm{C} \\
(\mathrm{N}) \\
\end{array}$ & $\begin{array}{c}\mathbf{t}_{\mathrm{pi}} \\
(\mathrm{ms}) \\
\end{array}$ & $\frac{t_{p f}}{(m s)}$ & $\begin{array}{c}\mathbf{v}_{\mathrm{p}} \\
(\mathrm{V}) \\
\end{array}$ & $n_{p}$ & Resultado \\
\hline I1 & Sem gás de & 10 & 10542 & - & - & - & - & NT \\
\hline $\mathrm{I} 2$ & purga & 15 & 10991 & 511561 & 18891430 & 0,42 & 2 & TR \\
\hline $\mathrm{I} 3$ & Com gás de & 10 & 10954 & 1050 & 2802 & 0,92 & 2 & TR \\
\hline I4 & purga & 15 & 10978 & 1971450 & 1971458 & 0,49 & 1 & TR \\
\hline
\end{tabular}

*Nota: E = Energia gerada no arco de soldagem; NT = Corpo de prova que não trincou e não apresentou sinal no SEA; TR = Corpo de prova que trincou e apresentou sinal do SEA.
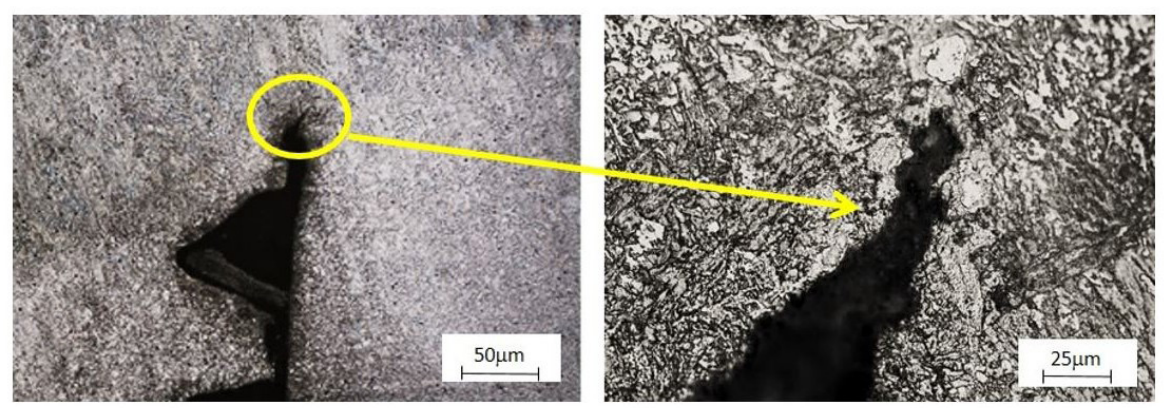

(a)

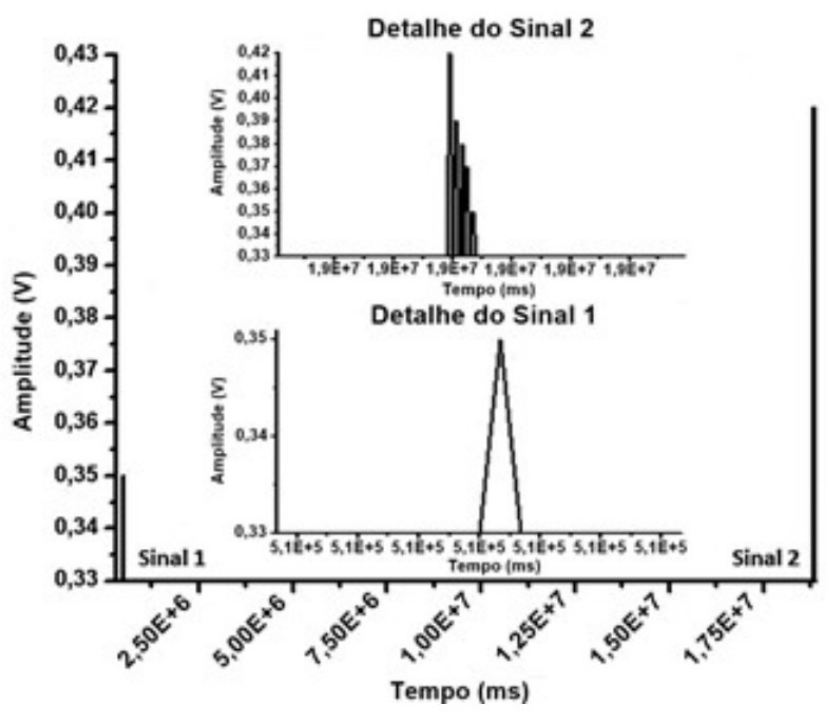

(b)

Figura 5. (a) Micrografia da TH na RGGZTA em I2; (b) Sinal capturado pelo SEA para I2.

$\mathrm{C}$ e as variáveis resultantes dos dados adquiridos pelo SEA são $t_{p i}, t_{p f}, v_{p}$ e $n_{p}$, sendo:

C - Carga registrada pela célula de carga no implante;

$\mathrm{t}_{\mathrm{pi}}$ - Tempo da primeira excitação (pico) registrada pelo SEA;

$\mathrm{t}_{\mathrm{pf}}-$ Tempo final registrado pelo SEA;

$\mathrm{v}_{\mathrm{p}}$ - Valor da maior excitação (amplitude máxima) registrada pelo SEA;

$\mathrm{n}_{\mathrm{p}}$ - Número de excitações (picos) armazenadas acima do limite de corte durante todo o ensaio.
Na Tabela 3, observa-se que o ensaio I1 não apresentou trinca, comprovado pela sua micrografia, e nem sinal registrado no SEA. O não surgimento da trinca, apesar da baixa energia de soldagem, pode ser explicado pela baixa tensão residual decorrente da baixa carga aplicada durante o ensaio. Segundo o estudo [4], é necessária uma carga mínima (de cerca de $10944 \mathrm{~N}$ ) aplicada no implante para que surja a trinca no CP.

O ensaio I2 apresentou trinca, Figura 5a, e captura de sinais pelo SEA, Figura $5 \mathrm{~b}$. Depois de transcorridos $8,53 \mathrm{~min}$ do início do ensaio, o SEA registrou o primeiro pico de $0,35 \mathrm{~V}$, indicando o surgimento da trinca. Após 314,86 min 
$(5,25 \mathrm{~h})$, houve o registro do segundo pico, com amplitude de $0,42 \mathrm{~V}$, indicando a propagação da $\mathrm{TH}$.

Observa-se na Figura 5a que a trinca foi gerada na fronteira da RGGZTA (região de grão grosseiro da ZTA)/ZF devido, provavelmente, à presença da restrição, ao tempo de propagação da trinca, ao hidrogênio residual decorrente da soldagem e à quantidade de carbono equivalente do metal de adição se encontrar na faixa de materiais susceptíveis. Essa trinca se propaga sem, entretanto, estender-se na direção da ZF, demonstrando ser uma TH. A trinca se apresenta de pequeno tamanho possivelmente devido à baixa tensão residual imposta pela solda.

$\mathrm{Na}$ soldagem do I3, foi utilizado gás de purga para a proteção da poça de fusão contra o ar atmosférico e, consequentemente, a ação do hidrogênio. O ensaio I3 apresentou uma trinca, Figura 6a, e captura de sinais pelo SEA, Figura $6 \mathrm{~b}$. Depois de transcorridos 1,05 segundos do início do ensaio, o SEA registrou o primeiro pico de $0,5 \mathrm{~V}$, indicando o surgimento da trinca. Após aproximadamente 3 segundos, houve o registro do segundo pico, com amplitude de $0,92 \mathrm{~V}$, que indica a propagação da $\mathrm{TH}$.
Assim como ocorrido no I2 e pela mesma justificativa, a trinca do ensaio I3 se formou na fronteira da RGGZTA/ZF, apresentando amplitude maior em um intervalo de tempo muito curto entre o primeiro e o último pico, demonstrando ser uma TH. A trinca se apresenta indicando que se propagaria na direção da ZF.

O I4, Figura 7a, foi soldado utilizando o gás de purga com a mesma finalidade do ensaio I3. Foi realizada a micrografia do implante e foram observados o aparecimento de uma trinca e a captura de sinais no SEA, Figura 7b. O pico surgiu após 33 minutos do início do ensaio com amplitude de $0,35 \mathrm{~V}$ e duração de $8 \mathrm{~ms}$, indicando uma microtrinca de hidrogênio. Mattioli [13], em seu estudo sobre fissuração de hidrogênio, utilizando teste implante em aço API 5L X70, também encontrou microtrincas de hidrogênio em seus ensaios.

Nos ensaios com gás de purga, ocorreram trincas independentemente da energia de soldagem. $O$ gás de purga protegeu a parte inferior do implante, entretanto, a face do cordão de solda ficou desprotegida porque o gás de proteção do consumível não foi satisfatório.
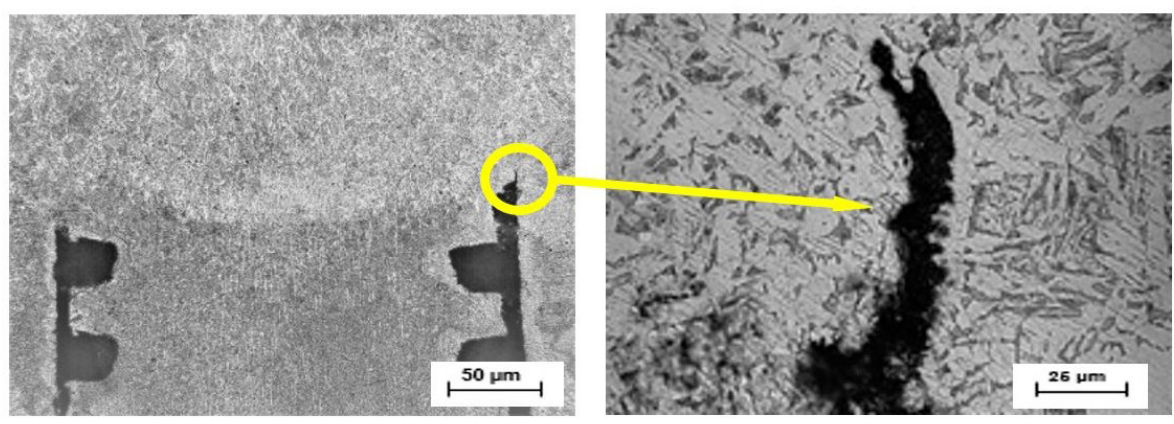

(a)

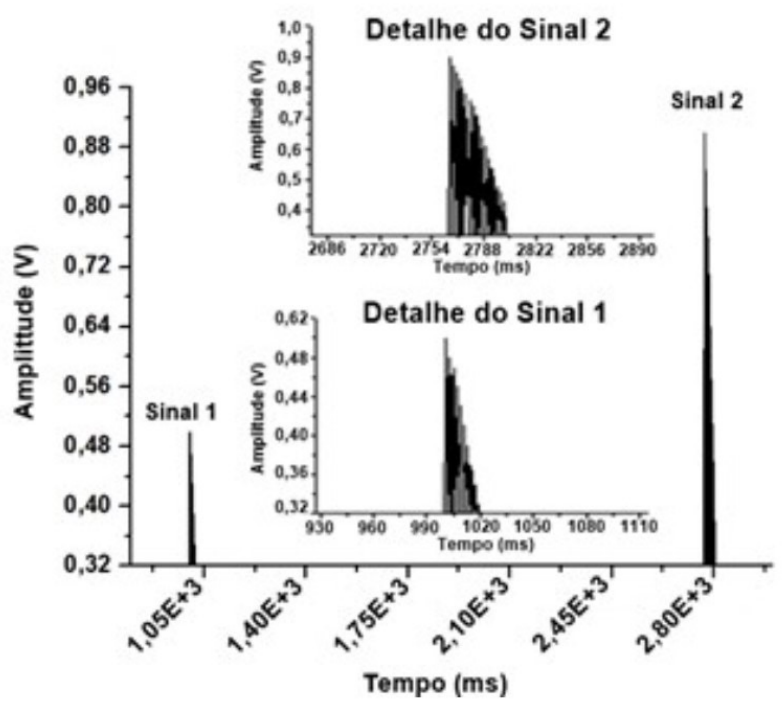

(b)

Figura 6. (a) Micrografia da TH na RGGZTA em I3; (b) Sinal capturado pelo SEA para I3. 

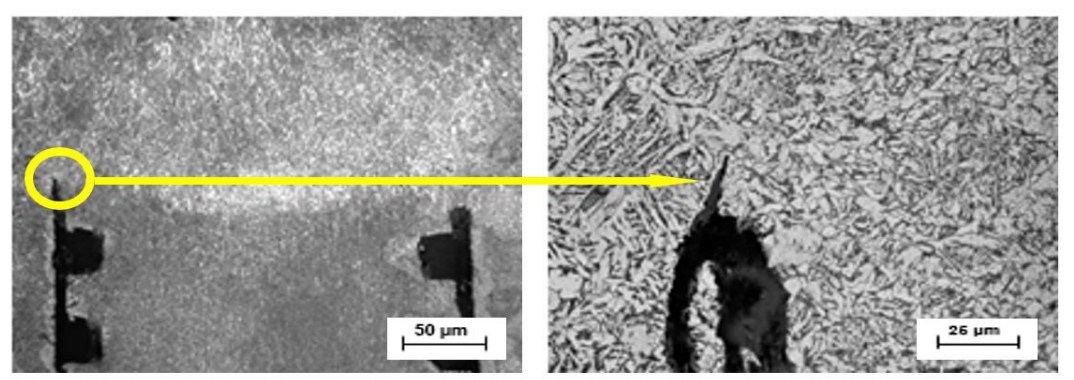

(a)

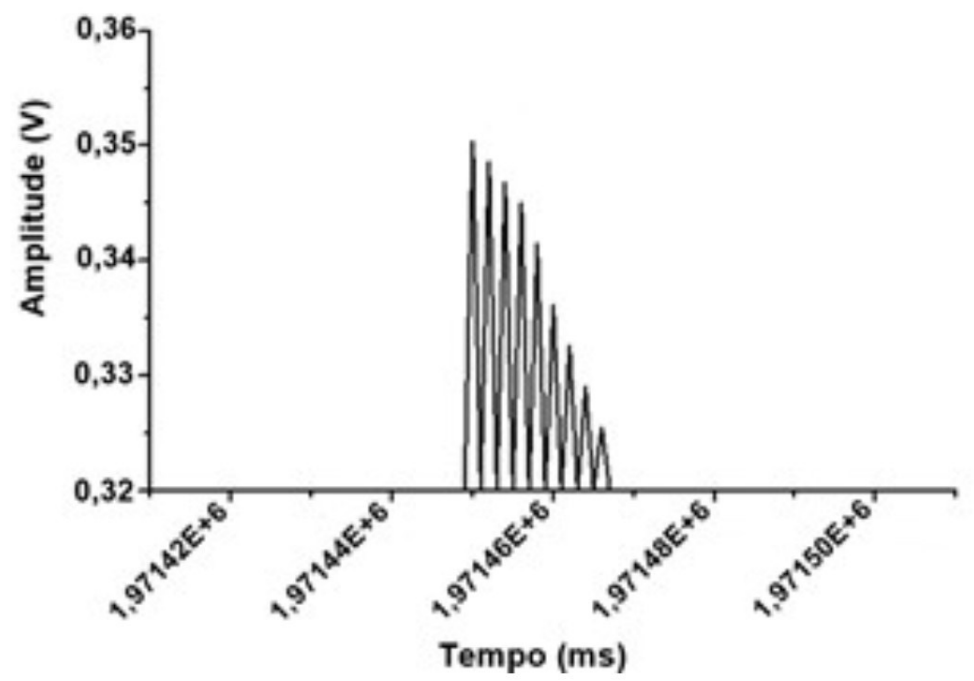

(b)

Figura 7. (a) Micrografia da TH na RGGZTA em I4; (b) Sinal capturado pelo SEA para I4.

A trinca de hidrogênio, frequentemente, origina-se a partir de concentradores de tensão, como na margem ou na raiz da solda, e podem ser micro ou macroscópicas. De acordo com Sharma e Maheshwari [8], essa trinca ocorre principalmente na ZTA ou na solda. Segundo Fals e Trevisan [3], a trinca de hidrogênio se forma sempre na região de alta concentração de tensões, localizada aproximadamente na linha de fusão, denominada de RGGZTA (área de maior fragilidade, pois é a zona de crescimento dos grãos da ZTA). A fissuração na ZF tem sido observada mais recentemente com a utilização de aços com menor carbono equivalente, mas com alta resistência mecânica, superior a $590 \mathrm{MPa}$.

Assim como no estudo de Fals e Trevisan [3], os sinais de EA obtidos nos ensaios realizados demonstraram que existe uma importante dependência entre os modos de fratura e as características dos sinais acústicos obtidos (amplitude), havendo também uma relação entre amplitude de sinais e carga aplicada. Assim, o sinal de EA aumentou à medida que a tensão aplicada diminuiu.

\subsection{Microdureza}

Na Figura 8, são apresentados os valores médios de microdureza Vickers (HV) na ZF, RGGZTA e no MB. Esse

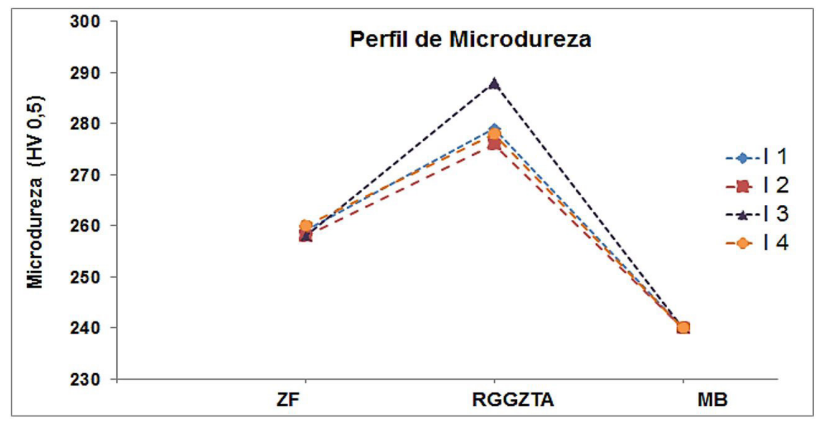

Figura 8. Perfil de microdureza em diferentes regiões dos ensaios I1, I2, I3 e I4.

comportamento de microdureza comprova que a dureza na RGGZTA é maior do que nas demais regiões.

Da análise do comportamento das microdurezas apresentadas nos ensaios, observa-se uma dureza média na RGGZTA de $276 \mathrm{HV}$ a $288 \mathrm{HV}$, na ZF de $258 \mathrm{HV}$ a $260 \mathrm{HV}$ e no MB sem muita variação da ordem de $240 \mathrm{HV}$. A partir da Tabela 4, observa-se um pequeno grau de dispersão nas medidas realizadas, atribuído a heterogeneidades estruturais em consequência de regiões que se recristalizaram ou não. 
Tabela 4. Valores de microdureza da ZF, RGGZTA e MB

\begin{tabular}{|c|c|c|c|c|c|c|c|c|c|c|c|}
\hline \multirow{2}{*}{$E(\mathrm{~kJ} / \mathrm{cm})$} & \multirow{2}{*}{ Teste } & \multirow{2}{*}{ Zona } & \multicolumn{7}{|c|}{ Pontos } & \multirow{2}{*}{ Média } & \multirow{2}{*}{$\begin{array}{l}\text { Desvio } \\
\text { Padrão }\end{array}$} \\
\hline & & & 1 & 2 & 3 & 4 & 5 & 6 & 7 & & \\
\hline \multirow[t]{4}{*}{10} & I1 & $\mathrm{ZF}$ & 258 & 256 & 265 & 259 & 264 & 261 & 252 & 259 & 4.5 \\
\hline & & RGGZTA & 276 & 279 & 302 & 278 & 288 & 283 & 268 & 279 & 10.8 \\
\hline & $\mathrm{I} 2$ & ZF & 257 & 260 & 262 & 254 & 258 & 261 & 255 & 258 & 3.0 \\
\hline & & RGGZTA & 266 & 269 & 286 & 278 & 272 & 276 & 283 & 276 & 7.3 \\
\hline \multirow[t]{5}{*}{15} & I3 & ZF & 257 & 260 & 255 & 256 & 260 & 258 & 261 & 258 & 2.3 \\
\hline & & RGGZTA & 267 & 288 & 292 & 304 & 288 & 281 & 309 & 288 & 14.0 \\
\hline & I4 & ZF & 260 & 251 & 257 & 261 & 258 & 270 & 262 & 260 & 5.8 \\
\hline & & RGGZTA & 268 & 271 & 284 & 287 & 270 & 280 & 278 & 278 & 10.8 \\
\hline & MB & & 237 & 240 & 244 & 251 & 232 & 239 & 245 & 240 & 6.1 \\
\hline
\end{tabular}

Comparando os perfis de microdureza, observa-se que os ensaios I1 e I3, realizados com menor energia de soldagem, apresentaram níveis de microdureza mais elevados, atribuídos às microestruturas de maior dureza formadas em consequência de maiores taxas de resfriamento, como no estudo de Cizek et al. [14] que apresentou um diagrama TRC (Transformação por Resfriamento Contínuo) de um aço API X80 para várias taxas de resfriamento. Nesse estudo [14], é mostrado que para taxas de resfriamento mais elevadas são formadas microestruturas com elevados níveis de microdureza, como a martensita.

O ensaio I3 apresentou a maior dureza, possivelmente em razão da menor energia de soldagem e da presença do gás de purga que interferiu na microdureza da região soldada, $o$ que influenciou na taxa de resfriamento e na microestrutura.

Segundo Silva [15], a microdureza está diretamente relacionada com o tempo de resfriamento da junta soldada, tipo de material e histórico de fabricação. O Estudo [15] sobre trincas de hidrogênio utilizando um aço ARBL obteve o mesmo comportamento de microdureza deste trabalho, com valores maiores na ZTA do que na ZF e MB.

\section{Conclusões}

Com base nos resultados obtidos neste trabalho, pode-se concluir que:

- O gás de purga não se mostrou eficiente na prevenção da trinca de hidrogênio nas condições previstas neste trabalho;
- A baixa carga inserida no ensaio I1, apesar da baixa energia de soldagem, evidenciou a necessidade de haver uma maior carga aplicada, concordando com a literatura, para que haja o surgimento da trinca no corpo de prova;

- Com os sinais obtidos pelo SEA, foi possível constatar a existência da trinca e, com os resultados da aquisição do sensor de emissão acústica, comprovar a viabilidade e sensibilidade desse sistema na detecção da TH. Dessa forma, foi possível observar o momento de formação e propagação da trinca de hidrogênio.

- A trinca de hidrogênio surgiu e se propagou em um intervalo de tempo máximo de ensaio de 5 horas.

- Verificou-se que a formação da TH ocorreu na região de grãos grosseiros da ZTA, o que se deve ao surgimento de microestruturas mais susceptíveis ao trincamento formadas durante o resfriamento.

\section{Agradecimentos}

Os autores deste trabalho agradecem ao Instituto Federal do Maranhão - IFMA pelo apoio ao desenvolvimento deste estudo, à Coordenação de Aperfeiçoamento de Pessoal de Nível Superior - CAPES pelo financiamento desta pesquisa com bolsa de estudo, e ao LTPSolda/IFMA pela realização dos ensaios.

\section{Referências}

1 Ohaeri E, Eduok U, Szpunar J. Hydrogen related degradation in pipeline steel: a review. International Journal of Hydrogen Energy. 2018;43:14584-14617.

2 Bezerra AA. Trinca a frio na soldagem. Sinergia. 2005;6:71-76.

3 Fals HC, Trevisan RE. Uso del ensayo de implante y la técnica de emisión acústica para estudiar el agrietamiento asistido por hidrógeno em la zona fundida en la soldadura de un acero HSLA-80. Revista de Metalurgia. 1999;35(4):233-241.

4 Martins WP. Utilização da técnica de emissão acústica no estudo da trinca de hidrogênio na soldagem de um aço de alta resistência [tese]. Uberlândia: Universidade Federal de Uberlândia; 2013. 
5 Ramos EJ, Guilherme LH. Caracterização da Soldagem Tig com Gás de Purga de Tubulação de Aço Inoxidável AISI 304 com e sem Deposição de Material. In: Anais do $21^{\circ}$ CBECIMAT - Congresso Brasileiro de Engenharia e Ciência dos Materiais; 2014 Nov 9-13; Cuiabá, Brasil. Cuiabá: Universidade Estadual Paulista; 2014.

6 Paiva TCC, Martins TRB, Souza GC, Pardal JM, Tavares SSM, Fonseca MC, et al. Análise da influência do gás de purga na raiz de juntas soldadas pelo processo GTAW em aço inoxidável superduplex UNS S32750. Soldagem e Inspeção. 2014;19:125-133.

7 Lagos SME, Fiallos DCC, Toasa JPG, Salinas CFP, Zumbana JAP. Influence of atmospheric oxygen content in the argon purge process in TIG welding in AISI 304 stainless steel pipe. Dyna (Bilbao). 2019;86:355-361.

8 Sharma SK, Maheshwari S. A review on welding of high strength oil and gas pipeline steels. Journal of Natural Gas Science and Engineering. 2017;38:203-217.

9 Silva HR, Ferraresi VA. Estudo dos Métodos de Determinação da Sensibilidade ao Trincamento à Frio em Juntas Soldadas. In: Anais do $16^{\circ}$ Simpósio de Pós-graduação em Engenharia Mecânica- Femec; 2006 Dez 11-13; Uberlândia, Brasil. Uberlândia: FEMEC; 2006.

10 Ferraresi VA. Estudo do fenômeno Trinca de Reaquecimento com auxílio da Emissão Acústica [tese]. Campinas: Universidade Estadual de Campinas; 1996.

11 Marques PV, Modenesi PJ. Algumas equações úteis em soldagem. Soldagem \& Inspeção. 2014;19:91-102.

12 Association Française de Normalisation - AFNOR. AFNOR NF A89-100: Fissuration à Froid Sur Implant. France: AFNOR; 1991. $11 \mathrm{p}$.

13 Mattioli CG, Modenesi PJ, Bracarense AQ, Oliveira MP. Fissuração pelo hidrogênio em um aço API 5L X70 soldado com eletrodos celulósicos de diferentes níveis de resistência. Soldagem e Inspeção. 2018;23:113-125.

14 Cizek P, Wynne BP, Hodgson PD, Muddle BC. Effect of simulated thermo mechanical processing on the transformation characteristics and microstructure of an X80 pipeline steel. In: Proceedings of the International Conference on Super- High Strength Steels; 2005 Nov 2-4; Rome, Italy. Rome: Associazione Italiana di Metallurgia and Centro Sviluppo Materiali; 2005.

15 Silva HR. Metodologia para análises de trinca de hidrogênio em soldas através de ensaios destrutivos e emissão acústica [dissertação]. Uberlândia: Universidade Federal de Uberlândia; 2010.

Recebido em: 31 Mar. 2020

Aceito em: 4 Set. 2020 